Mathematical Modelling and AnAlysis

Volume 17 Number 1, February 2012, 21-30

http://dx.doi.org/10.3846/13926292.2012.644046

(c) Vilnius Gediminas Technical University, 2012
Publisher: Taylor\&Francis and VGTU

http://www.tandfonline.com/TMMA

Print ISSN: 1392-6292

Online ISSN: 1648-3510

\title{
Segmenting the Eye Fundus Images for Identification of Blood Vessels
}

\section{Gediminas Balkys and Gintautas Dzemyda}

\author{
Institute of Mathematics and Informatics, Vilnius University \\ Akademijos 4, LT-08663 Vilnius, Lithuania \\ E-mail(corresp.): Gediminas.Balkys@mif.vu.lt \\ E-mail: Gintautas.Dzemyda@mii.vu.1t
}

Received August 31, 2010; revised September 15, 2011; published online February 1, 2012

\begin{abstract}
Retinal (eye fundus) images are widely used for diagnostic purposes by ophthalmologists. The normal features of eye fundus images include the optic nerve disc, fovea and blood vessels. Algorithms for identifying blood vessels in the eye fundus image generally fall into two classes: extraction of vessel information and segmentation of vessel pixels. Algorithms of the first group start on known vessel point and trace the vasculature structure in the image. Algorithms of the second group perform a binary classification (vessel or non-vessel, i.e. background) in accordance of some threshold. We focus here on the binarization [4] methods that adapt the threshold value on each pixel to the global/local image characteristics. Global binarization methods [5] try to find a single threshold value for the whole image. Local binarization methods [3] compute thresholds individually for each pixel using information from the local neighborhood of the pixel. In this paper, we modify and improve the Sauvola local binarization method [3] by extending its abilities to be applied for eye fundus pictures analysis. This method has been adopted for automatic detection of blood vessels in retinal images. We suggest automatic parameter selection for Sauvola method. Our modification allows determine/extract the blood vessels almost independently of the brightness of the picture.
\end{abstract}

Keywords: image analysis, binarization, retinal images, eye fundus, blood vessels identification.

AMS Subject Classification: $62 \mathrm{H} 35$.

\section{Introduction}

Image processing techniques find application in all modern fields of medical science. Retinal images are widely used for diagnostic purposes by ophthalmologists [6]. The extracted blood vessels are used for automatic retinal image registration [2]. The structure of vasculature can be exploited for automated human identification purpose also [5]: here it is very important to extract blood vessels from the retinal image precisely. 
The focus of this paper is to detect and extract correctly blood vessels from eye fundus image.

Retinal images are acquired with a digital fundus camera. The type of the camera allows us to get the images of high or poor quality. In this paper we consider analysis of pictures not of the best quality and large size: their size is $768 \times 512$ that is often used by ophthalmologists. It may be presumed that the higher picture size allows better results but it needs for much faster analysis methods.

Usually eye fundus images are color. They can be analyzed either as they stand in RGB format or converted to a gray scale $\{0, \ldots, 255\}$, and then binarized, i.e. converted to $\{0,1\}$. The binarization is used in this paper when foreground is vasculature, its pixels take value 1. Some threshold divides foreground and background when binarization is performed. Here we deal with the thresholding problem that is directly related to the binarization problem. This is a general problem that arises in various applications, e.g. scanned document analysis. Example of grey scale eye fundus picture and its binarization are presented in Figure 1.

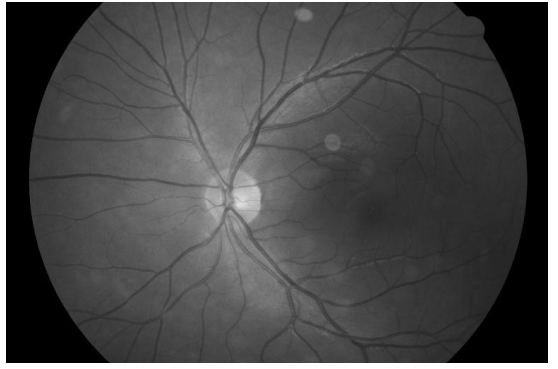

(a)

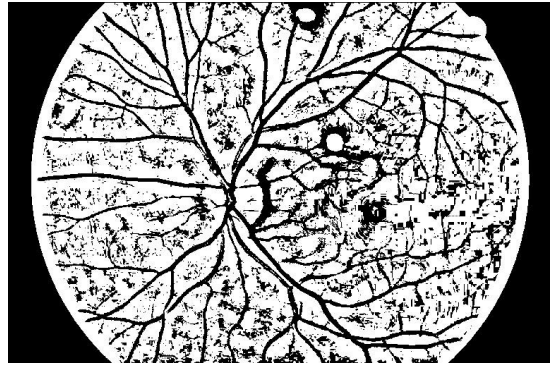

(b)

Figure 1. Identifying the blood vessels: (a) original image, (b) extracted vasculature using standard Sauvola method.

We can categorize general purpose image thresholding methods [4]:

1. Histogram shape-based methods, where valleys and curvatures of the smoothed histogram are analyzed;

2. Clustering-based methods, where the gray level samples are clustered in two parts as background and foreground, or alternately are modeled as a mixture of two Gaussians;

3. Entropy-based methods result in algorithms that use the entropy of the foreground and background region, the cross-entropy between the original and binarized images;

4. Object attribute-based methods search a measure of similarity between the gray-level and the binarized images, such as fuzzy shape similarity or edge coincidence; 
5. Spatial methods use higher-order probability distribution and/or correlation between pixels;

6. Global/local methods adapt the threshold value on each pixel to the global/local image characteristics.

On the one hand, global thresholding-based binarization (we call it global binarization) tries to find a single threshold value for the whole image. Global binarization methods are very fast and they give good results for scanned text documents where background and foreground is uniformly different in the whole image (for example, in documents where letters are black and foreground is grey). One of the global binarization methods is the Otsu method. It is very fast, but if we use a single threshold value for all images, we can lose very important information about blood vessels. Unfortunately, the Otsu method can give good result only for images where vasculature is sharp in the whole image.

On the other hand, local binarization methods try to overcome this problem by computing thresholds individually for each pixel using information from the local neighborhood of the pixel. The computational expenditure of the local binarization methods depends on the number of analysed local neighbours of the pixel. Therefore, local binarization methods are slower than global binarization methods, but they are more exact.

The aim of this paper is to critically analyse the practice of the detecting blood vessels in retinal images using global/local statistics. We analyze the local Sauvola method here. This method has been modified and improved by extending its abilities to be applied for eye fundus pictures analysis, and adopted for automatic detection of blood vessels in retinal images.

\section{Methodology}

The identification of blood vessels consists of four steps:

1. Converting the color retinal image to the grey scale.

2. Computing a threshold $t(x, y)$ for pixel $(x, y)$.

3. Binarization of the image.

4. Noise removing.

In the first step, the color retinal image has to be converted to grey scale using the standard conversion: grey $=0.3 R+0.59 G+0.11 B$, where $R, G, B$ represents the colors red, green and blue respectively, with values from 0 to 255 [7]. Grey scale images in which $g(x, y) \in[0,255]$ are the intensity of pixel $(x, y)$. The aim is to compute a threshold $t(x, y)$ for each pixel and to convert the grey scale image into the binary image with the intensity function $b(x, y)$ :

$$
b(x, y)= \begin{cases}1, & \text { if } g(x, y) \leqslant t(x, y) \\ 0, & \text { otherwise }\end{cases}
$$


It is difficult to achieve consistent quality with a fixed threshold $t(x, y)$ for all pixels while processing retinal images, because both foreground and background colors vary significantly among parts of image. We will pay attention to local methods, particularly to the local Sauvola method. The reason of selecting this method is its better performance in analysis of scanned text images that, as eye fundus images, have both light places and dark shadows [7].

In Section 3, the details of Sauvola method are discussed. In Section 4, the suggestions to improve its efficiency are presented. Binarization is examined experimentally in Section 5. Noise removing is discussed in Section 6. In Section 7, the problems on speedup of computing a threshold $t(x, y)$ are discussed.

\section{$3 \quad$ Local Thresholding using Sauvola Method}

Various adaptive methods have been proposed for binarization of the image [1]. One of them is Niblack's method [3]:

$$
t(x, y)=\mu(x, y)+k \sigma(x, y)
$$

where $\mu(x, y)$ and $\sigma(x, y)$ are local mean and local standard deviation, respectively, computed of pixel intensities in $w \times w$ window centered around the pixel $(x, y)$, and $k$ is a parameter that takes positive value from 0 to 1 .

Niblack's method usually is used for scanned text documents. We can split scanned text document into small blocks (e.g. we can detect lines, words or letters) and each block can be binarized. For scanned text binarization, we can select static $k$ value and get sufficiently good results, because foreground and background are significantly different in many documents. It is not possible to split the retinal image into small blocks, because all blood vessels are connected. Each retinal image is of different intensity. Therefore, it is very important to choose the proper parameters for each image. In the Niblack method, the noise that is presented in the background remains dominant in the final binary image. Consequently, if the objects are sparse in an image, a lot of background noise will be left. Sauvola and Pietikainen [3] modified Niblack's method. They have proposed a method (it is named Sauvola's method) that solves this problem by adding a hypothesis on the gray values of text and background pixels (text pixels have gray values near 0 and background pixels have gray values near 255), which results in the following formula for the threshold:

$$
t(x, y)=\mu(x, y)\left[1+k\left(\frac{\sigma(x, y)}{R}\right)\right],
$$

where $R$ is the maximum value of the standard deviation.

The idea of Niblack's and Sauvola's methods is to vary the threshold over the image, based on the parameters $\mu(x, y), \sigma(x, y), k$ and $R$ for a fixed size window $w \times w$. In our case, the window $w \times w$ must be chosen large enough to include retinal structures and the background.

The smaller value of $k$, the thicker is the binarized vessel and the more wide overlap exists between blood vessels. This is essential when we try to disclose a 
whole connected vasculature. A smaller sliding windows size $w \times w$ will produce thinner strokes. Optimal combination of $k$ and the size of the sliding window would produce a good binary image. Unfortunately, each image has individual optimal values of these parameters.

\section{Thresholding Based on Retinal Images Global/Local Statistics}

Our destiny is that parameters be selected automatically to get good binarization result for each image. In this paper, we confine on automated selection of parameter $k$ for Sauvola method.

We suggest three-step algorithm for $k$ selection:

1. Finding $k$, global for the whole image: $k^{\prime}=\sigma^{\prime} / \mu^{\prime}$.

2. Finding $k$, local for sliding window of size $w \times w$ centered around the pixel $(x, y)$ :

$$
k^{\prime \prime}(x, y)=\sigma^{\prime \prime}(x, y) / \mu^{\prime \prime}(x, y) .
$$

3. Defining the adaptive value of $k$ :

$$
k(x, y)=(1-e) k^{\prime}+e k^{\prime \prime}(x, y)
$$

Here $\mu^{\prime}$ and $\sigma^{\prime}$ are global mean and global standard deviation, respectively, computed of pixel intensities in the whole image, $\mu^{\prime \prime}(x, y)$ and $\sigma^{\prime \prime}(x, y)$ are local mean and local standard deviation computed of pixel intensities in $w \times w$ window centered around the pixel $(x, y) ; e \in[0,1]$ is some parameter that defines ratio between global and local decision when choosing the value of $k$ for the analyzed image

The value of $e$ depends on the image type (retinal image, scanned text, satellite images, etc.). If $e$ value is $0, k$ value depends entirely on the whole image pixel intensities. If $e$ value is $1, k$ value depends on pixel intensities in $w \times w$ window centered around the pixel $(x, y)$. In further experiments, $e$ value has been chosen equal to 0.5 seeking to keep the same influence of global and local properties of the image.

\section{Vasculature Extraction}

In this paper, we modify Sauvola's method for retinal images analysis by introducing a possibility of automated selection of parameter $k$. The modified method allows to determine/extract the blood vessels almost independently of the brightness of the picture. This is illustrated by experiments in Figure 2. Two images are analyzed: dark (Figure 2-left) and bright (Figure 2-right).

Images, converted to grey scale, are given in Figure 2(a). The size of images is $768 \times 512$ pixels. Application of the original Sauvola method, using recommended in [3] value 0.34 , is illustrated by Figure 2(b). In both cases, we see a lot of noise that makes troubles in discovering the blood vessels. 

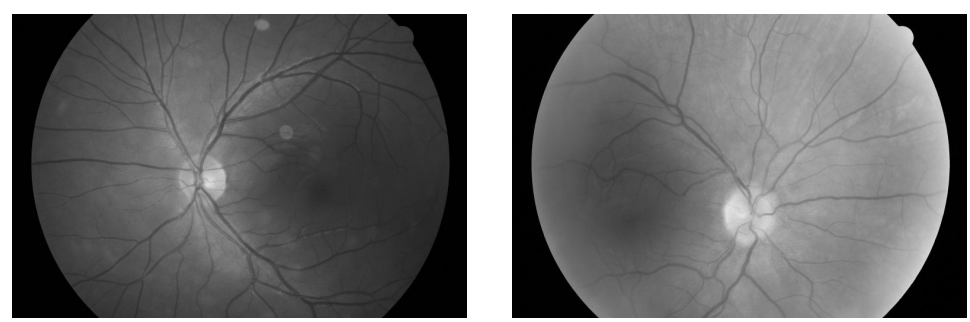

(a)
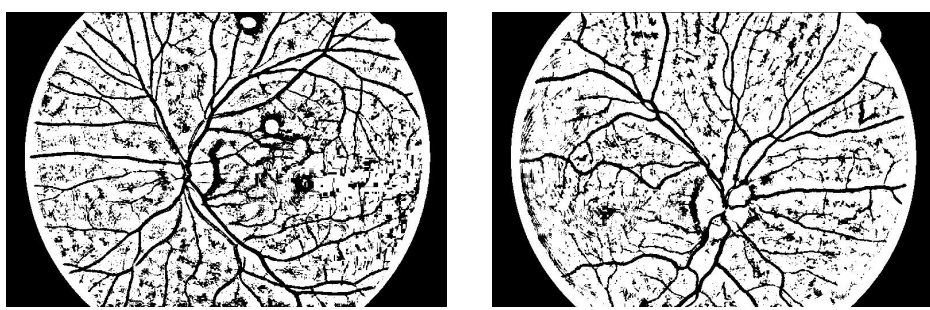

(b)
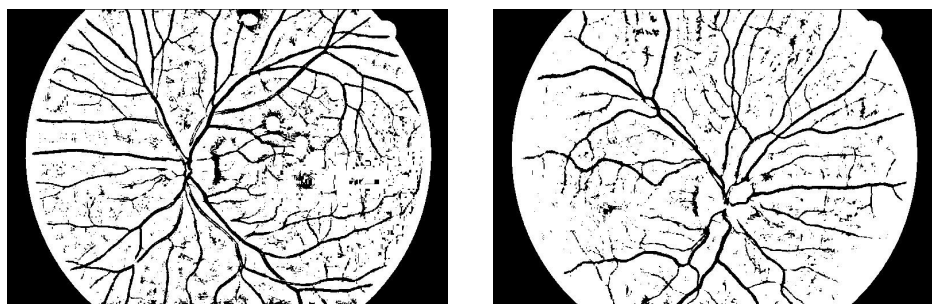

(c)

Figure 2. (a) Grey scale images, (b) binarization by the original Sauvola method, (c) binarization by the modified Sauvola method.

The results of the modified Sauvola method are given in Figure 2(c), here $R=128$ (such is a maximum value for standard deviation). The size of the window $w \times w$ is $15 \times 15$, because the maximum width of blood vessels in our eye fundus image $(768 \times 512$ pixels resolution $)$ is 10 pixels. If we have bigger image resolution, we can proportional increase $\mathrm{w}$ value automatically.

Figure 2 shows that application of the Sauvola method with automatic $k$ parameter selection allows determining/extracting blood vessels with less noise and less influence of the brightness as compared with the original Sauvola method.

\section{Noise Removing}

Removal of noises is a very important step in the image analysis. Our aim is to determine a vasculature. Regarding the specificity of the problem that the blood vessels must be connected, two rules are possible to remove the noise 
from binarized images:

1. Delete all unconnected pixels.

2. Remove objects with a thickness of more than thickest blood vessel.

Under the last rule we can delete the fovea that is a part of the eye located in the center of the retina.
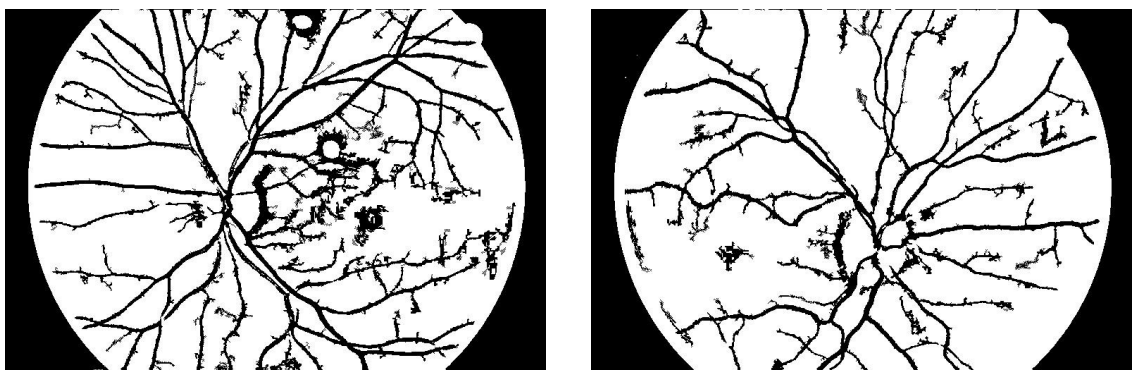

(a)
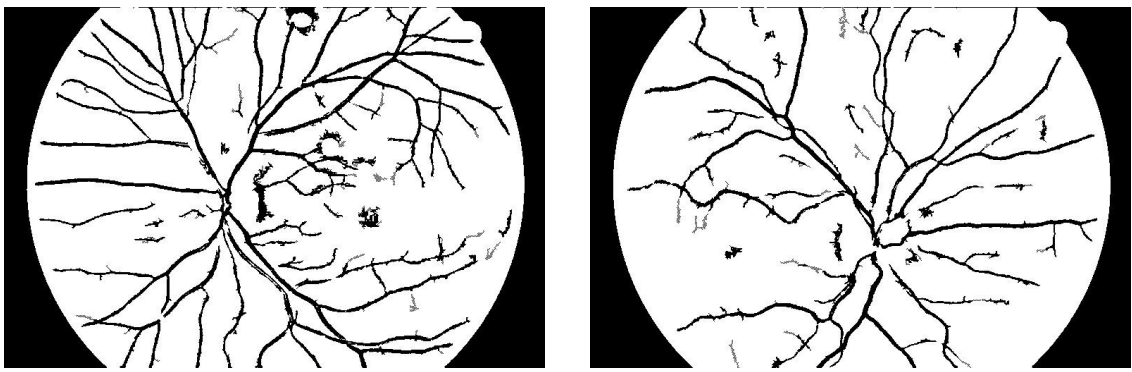

(b)

Figure 3. Vasculature with removed noise: (a) using original Sauvola method, (b) using modified Sauvola method.

Figure 3 illustrates experiments on noise removing using original Sauvola's method (Figure 3(a)) and the modified method (Figure 3(b)). The modified Sauvola method gives much clearer view of vasculature.

Small unconnected blood vessels may be lost when removing unconnected pixels. For image analysis and registration, these vessels are not very important, because for image registration we must skeletonize blood vessels. Image registration is the process of overlaying images (two or more) of the same scene taken at different times, from different viewpoints, and/or by different sensors $[1,6]$. Thickness of the skeletonized blood vessel is one pixel. Regardless to the real thickness of a blood vessel, all vessels become equally important after skeletonization.

The modified Sauvola method have more numerous discontinuities and it can look to be worse than the original Sauvola method. But if we look more carefully, we see, that the modified method detects less incorrect blood vessels. If we have less incorrect blood vessels, we can make a better image registration 
and human identification. In these examples we use the same noise removing algorithm. For the modified method we can use less aggressive noise removing algorithm and to get more blood vessels pixels.

\section{Speedup in Computing a Threshold}

For each pixel, the evaluation of a threshold $t(x, y)$ is computationally expensive. For example, the image size $768 \times 512$ means that the image contains about 0.4 mega pixels. If the image is larger, e.g. $1920 \times 1080$ pixels, its size grows significantly till about 2 million pixels.

In this section, two possibilities to speed up the computations of a threshold $t(x, y)$ are discussed:

1. Calculations of threshold $t(x, y)$ for neighboring pixels.

2. Calculation of local mean $\mu(x, y)$ and local standard deviation $\sigma(x, y)$.

When calculating the threshold $t(x, y)$ for pixel $(x, y)$, the assumption that 8 neighboring pixels $(x-1, y),(x+1, y),(x, y-1),(x, y+1),(x-1, y-1)$, $(x-1, y+1),(x+1, y-1),(x+1, y+1)$ have the same threshold may be done. This allows saving computing time several times and increasing the size of local sliding window $w \times w$, remember that this local window must be large enough to include retinal structures and the background. The extracted vasculature (including noise removing) is presented in Figure 4: (a) without optimization, (b) with optimization.

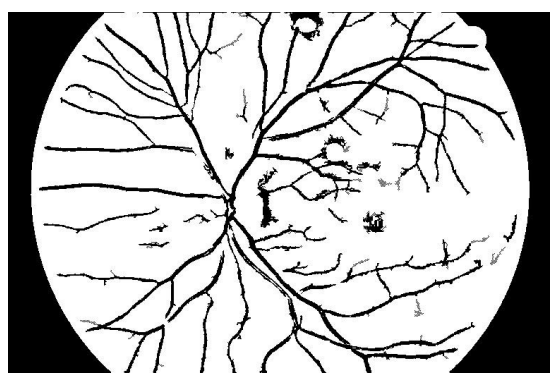

(a)

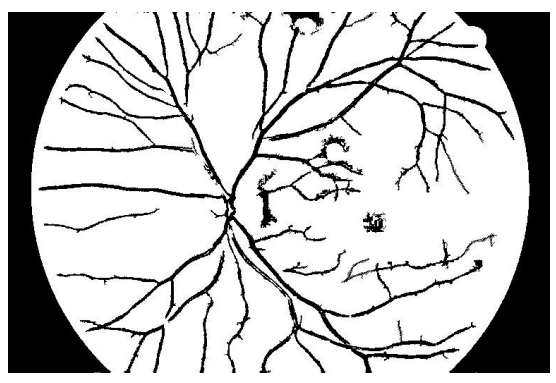

(b)

Figure 4. Calculating the threshold: (a) without optimization, (b) with optimization.

Optimization resulted in saving computing time but loosing thin vessels. However, the general vessel structure remains the same.

We note that for calculation of the local mean $\mu(x, y)$ and local standard deviation $\sigma(x, y)$, not all $w \times w$ pixels of the local sliding window may be used. One possible scheme of used pixels (they are marked in black) is given in Figure 5. This allows to optimize computations of the threshold, too.

The extracted vasculature including noise removing is presented in Figure 6: (a) using all $w \times w$ pixels, (b) using pixels by scheme in Figure 5. Like above, such an optimization resulted in saving computing time but loosing thin vessels. However, the general vessel structure remains the same. 


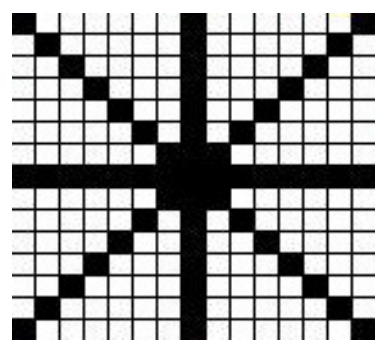

Figure 5. Scheme of used pixels in the local sliding window.

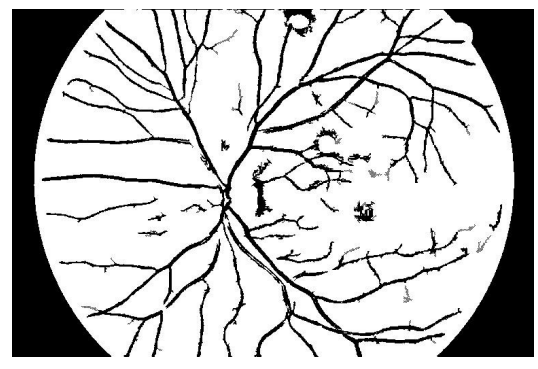

(a)

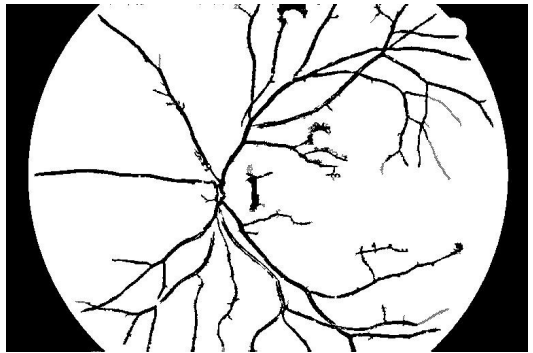

(b)

Figure 6. Extracted vasculature: (a) using $w \times w$ pixels, (b) using pixels by scheme in Figure 5.

\section{Conclusions}

In this paper, we modify and improve the Sauvola local binarization method by extending its abilities to be applied for eye fundus picture analysis. This method has been adopted for automatic detection of blood vessels in retinal images. We suggest automatic parameter selection for Sauvola method. Our modification allows determine/extract the blood vessels almost independently of the brightness of the picture with less noise.

Two rules are suggested to remove the noise from binarized images. The modified Sauvola method gives much clearer view of vasculature after noise removing as well.

Two possibilities to speed up the computing of a threshold are disclosed. In both cases, it is possible to save the computing time several times and increasing the size of local sliding window, if necessary. Thin vessels may be lost, but the general vessel structure remains the same.

Future research may be oriented to the automation of selecting the size of the local sliding window. Data for such research should be the eye fundus images exclusively. 


\section{References}

[1] B. Gatos, I. Pratikakis and S.J. Perantonis. Adaptive degraded document image binarization. Pattern Recognition, 39:317-327, 2006. http://dx.doi.org/10.1016/j.patcog.2005.09.010.

[2] M. Meilunas, A. Ušinskas, R. Kirvaitis and R.A. Dobrovolskis. Automatic contouring of segmented human brain ischemic stroke region on ct images. Math. Model. Anal., 8(1):43-50, 2003. http://dx.doi.org/10.1080/13926292.2003.9637209.

[3] J. Sauvola and M. Pietikainen. Adaptive document image binarization. Pattern Recognition, 33(2):225-236, 2000.

http://dx.doi.org/10.1016/S0031-3203(99)00055-2.

[4] M. Sezgin and B. Sankur. Survey over image thresholding techniques and quantitative performance evaluationl. Journal of Electronic Imaging, 13(2):146-165, 2004. http://dx.doi.org/10.1117/1.1631315.

[5] P. Treigys, G. Dzemyda and V. Barzdžiukas. Automated positioning of overlapping eye fundus images. Computational Science - ICCS 2008, Lecture Notes in Computer Science, LNCS 5101:770-779, 2008.

http://dx.doi.org/10.1007/978-3-540-69384-0_82.

[6] P. Treigys, V. Šaltenis, G. Dzemyda, V. Barzdžiukas and A. Paunksnis. Automated optic nerve disc parameterization. Informatica, 19(3):403-420, 2008.

[7] B. Zitova and J. Flusser. Image registration methods: a survey. Image and Vision Computing, 21:977-1000, 2003.

http://dx.doi.org/10.1016/S0262-8856(03)00137-9. 